http://dx.doi.org/10.11646/phytotaxa.162.5.1

\title{
A revision of the genus Menonvillea (Cremolobeae, Brassicaceae)
}

\author{
DIEGO L. SALARIATO ${ }^{1,3}$, FERNANDO O. ZULOAGA ${ }^{1}$ \& IHSAN A. AL-SHEHBAZ ${ }^{2}$ \\ ${ }^{1}$ Instituto de Botánica Darwinion, Labardén 200, Casilla de Correo 22, B1642HYD, San Isidro, Buenos Aires, Argentina \\ ${ }^{2}$ Missouri Botanical Garden, P.O. Box 299, St. Louis, MO 63166-0299, U.S.A. \\ ${ }^{3}$ Author for correspondence: Diego L. Salariato,dsalariato@darwin.edu.ar
}

\begin{abstract}
Following the most recent phylogenetic analyses of Menonvillea, an updated taxonomic revision of the genus based on molecular and morphological data is presented here. Menonvillea currently includes 24 species distributed in Argentina and Chile. Three new sections, sects. Cuneata, Menonvillea, and Scapigera, are proposed. One subspecies is raised to the specific rank, and the new combination $M$. marticorenae is proposed. Descriptions, keys to all taxa, updated geographical distributions, maps, and illustrations are provided. Lectotypes for M. filifolia, M. minima, M. purpurea, and M. flexuosa f. tomentosa are designated.
\end{abstract}

\section{Resumen}

Sobre la base de los últimos estudios filogenéticos de Menonvillea, se presenta una revisión taxonómica basada en datos moleculares y morfológicos. Como resultado, Menonvillea incluye actualmente 24 especies distribuidas en Argentina y Chile. Tres nuevas secciones dentro del género son propuestas: Sect. Cuneata, Sect. Menonvillea, y Sect. Scapigera. Una subespecie es elevada al rango específico, y la nueva combinación M. marticorenae es aquí propuesta. Sinónimos, descripciones y claves para todos los taxones son provistos. La distribución actualizada de los taxones con sus mapas correspondientes son presentados, y varias especies son ilustradas. Adicionalmente lectotipos para M. filifolia, M. minima, M. purpurea, y M. flexuosa f. tomentosa también son designados.

Key words: Andes, Argentina, Chile, Cruciferae, taxonomy

\section{Introduction}

Menonvillea de Candolle (1821a: 236) is a genus of Brassicaceae (Cruciferae) distributed mainly along the Andes of Argentina and Chile, with some taxa growing in southern Patagonia (Santa Cruz Province in Argentina southward into Región Magallanes y Antarctica in Chile) and others restricted to the Antofagasta desert in northern Chile (Al-Shehbaz, 2008, 2010; Al-Shehbaz \& Marticorena, 1990).

Several taxa of Menonvillea were originally described in the genera Hexaptera Hooker (1830: 350), Dispeltophorus Lehmann (1832: 7), Decaptera Turczaninow (1846: 497), and Cymatoptera Turczaninow (1854: 309). Hooker (1830) included in Hexaptera taxa with 3-winged fruit valves (two lateral wings and one dorsal) and assigned to Menonvillea species with 2-winged valves (only two lateral wings). By contrast, Turczaninow (1846) included in Decaptera taxa with 5-winged fruit valves. Both Cymatoptera and Dispeltophorus have 2-winged valves and therefore do not differ from Menonvillea sensu Hooker (1830). Rollins (1955) monographed Menonvillea to include 29 species, placed the above-mentioned four genera in its synonymy, and indicated that the fruit wing number is variable and unreliable to delimit genera. He characterized Menonvillea by having schizocarpic silicles that break at maturity into two, 1-seeded, indehiscent, 2-, 3-, or 5-winged (or rarely wingless) mericarps. Al-Shehbaz (2008) accepted 23 species in the genus and later (Al-Shehbaz, 2010) described $M$. zuloagaensis Al-Shehbaz. 
basal leaves, and coarsely papillate petals and stamens bases support the relationship of $M$. zuloagensis to $M$. scapigera and M. famatinensis. Although this species was not sequenced for the molecular analysis of Salariato et al. (2013), morphological characters analyzed herein support its placement in Sect. Scapigera.

\section{Acknowledgments}

Funding of this work was provided by CONICET (Consejo Nacional de Investigaciones Científicas y Técnicas) grants PID 11220100100207 and PIP 11220100100155 and National Geographic Society grants \#8365-07 and \#8862-10, for which we are profoundly grateful. Fieldwork and visits to herbaria was supported by the Myndel Botanical Foundation grants in 2010, 2011, and 2012. We are profoundly grateful to the directors, curators, and collection managers of the following herbaria: B, BAA, BAB, BH, BM, BCRU, CONC, CORD, E, F, G, GH, K, KW, LE, LIL, LP, MEL, MERL, MO, NY, P, PR, S, SGO, SI, SRFA, U, UC, UPS, US. We thank Franscisco Rojas (IBODA) for preparing the illustrations and Fabian Tricarico Museum of Natural Sciences "Bernardino Rivadavia" for his help in the scanning electron microscopy. Finally, our special thanks go to Natalia Álvarez for collaboration during the first stages of this work.

\section{References}

Al-Shehbaz, I.A. (2006) New or Noteworthy taxa of Argentinean and Chilean Brassicaceae (Cruciferae). Darwiniana 44(2): 359-362.

Al-Shehbaz, I.A. (2008) Brassicaceae. In: Zuloaga, F.O., Morrone, O., Belgrano, M.J., (eds.) Catalogue of the vascular plants of the southern cone (Argentina, southern Brazil, Chile, Paraguay and Uruguay), vol 2: Dicotyledoneae: AcanthaceaeFabaceae (Abarema-Schizolobium). Monographs in Systematic Botany from the Missouri Botanical Garden 107: 16631709.

Al-Shehbaz, I.A. (2010) Menonvillea zuloagaensis and Mostacillastrum hunzikeri (Brassicaceae), two new species from Argentina. Darwiniana 48(1): 59-63.

Al-Shehbaz, I.A. \& Marticorena, C. (1990) Menonvillea rollinsii (Brassicaceae), a new shrubby species from Chile. Journal of the Arnold Arboretum 71: 135-138.

Al-Shehbaz, I.A., Muñoz-Schick, M. \& Morales, V. (2011) The present status of Brassicaceae taxa described by Rodulfo and Federico Philippi. Harvard Papers in Botany 16: 279-291. http://dx.doi.org/10.3100/0.25.016.0204

Boelcke, O. (1951) Una nueva especie de "Hexaptera" de la Rioja. Revista Argentina de Agronomía 18(3): 171-175.

Brown, R. (1826) Botanical appendix. In: Denhamn, D. \& Clapperton, H (eds.) Narrative of travels and discoveries in Northern and Central Africa, in the years 1822, 1823, and 1824, by Major Denham, Captain Clapperton, and the late Dr. Oudney. Cummings, Hilliard \& Co., Boston, pp. 61-92.

Cabrera, A.L. \& Willink, A. (1973) Biogeografía de América Latina, Serie Biológica, Monografía 13. Organización de los Estados Americanos, Washington, D.C., 117 pp.

Candolle, A.P. de (1821a) Mémoire sur la famille des Crucifères. Mémoires du Muséum d'Histoire Naturelle 7: 169 -252.

Candolle, A.P. de (1821b) Regni vegetabilis systema naturale, sive ordines, genera et species plantarum secundum methodi naturalis normas digestarum et descriptarum, vol. II. Sumptibus sociorum Treuttel et Würtz, Paris, 745 pp. http://dx.doi.org/10.5962/bhl.title.59874

Dusén, P. (1900) Die Gefässpflanzen der Magellansländer; nebst einem Beitrage zur Flora der Ostküste von Patagonien. Svenska expeditionen till Magellansländerna 3(5): 77-266. http://dx.doi.org/10.5962/bhl.title.9040

Fischer, F.E.L. \& Meyer C.A. (1835) Index seminum, quae Hortus Botanicus Imperialis Petropolitanus pro mutua commutatione offert: accedunt Animadversiones botanicae nonnullae. Petropoli, St. Petersburg, 42 pp.

Gay, C. (1846) Flora Chilena, tomo I. Fain \& Thunot, Paris, 493 pp.

Gilg, E. \& Muschler R. (1909) Aufzählung aller zur Zeit bekannten südamerikanischen Cruciferen. Botanische Jahrbücher für Systematik, Pflanzengeschichte und Pflanzengeographie 42: 437-487.

Hastings, R.I. (1905) Observations on the flora of central Chile. Bulletin of the Torrey Botanical Club 32: 613-623.

Hooker, W.J. (1830) On a New Genus of Plants of the Nat. Ord. Cruciferae, from the Andes of Chili and Mendoza. Botanical Miscellany 1: 349-352.

Hosseus, C.C. (1921) Flora Argentina. Estudios comparativos sobre la vegetación de las provincias de La Rioja y San Juan. Boletin de la Academia Nacional de Ciencias de Cordoba 26: 5-160.

Jackson, B.D. (1894) Index Kewensis plantarum phanerogamarum nomina et synonyma omnium generum et specierum a 
Linnaeo usque ad annum MDCCCLXXXV complectens nomine recepto auctore patria unicuique plantae subjectis, vol II. Oxford, Clarendon Press, 1299 pp.

http://dx.doi.org/10.5962/bhl.title.66720

Johnston, I.M. (1929a) Papers on the Flora of Northern Chile: The coastal flora of the departments of Chañaral and Taltal. Contributions from the Gray Herbarium of Harvard University 85: 1-137.

Johnston, I.M. (1929b) Papers on the Flora of Northern Chile: Undescribed species from the cordillera of Atacama. Contributions from the Gray Herbarium of Harvard University 85: 164-172.

Khanna, K.R. \& Rollins, R.C. (1965) A taxonomic revision of Cremolobus (Cruciferae). Contributions from the Gray Herbarium of Harvard University 195: 135-157.

Kuntze, O. (1898) Revisio generum plantarum: vascularium omnium atque cellularium multarum secundum leges nomenclaturae internationales cum enumeratione plantarum exoticarum in itinere mundi collectarum, vol. $3 b$. A. Felix, Leipzig, 576 pp. http://dx.doi.org/10.5962/bhl.title.327

Lehmann, J.G.C. (1832) Delectus Seminum quae in horto Hamburgensium botanico e collectione anni 1832 mutuae commutationi offeruntur. Typis Ioannis Augusti Meissneri, Hamburg, 7 pp.

Morrone, J.J. (2001) Biogeografía de América latina y el Caribe. M\&T-Manuales \& Tesis SEA, Zaragoza, 148 pp.

Nordenstam, B. (1980) The Herbaria of Lehmann and Sonder in Stockholm, with Special Reference to the Ecklon and Zeyher Collection. Taxon 29: 279-288. http://dx.doi.org/10.2307/1220289

Philippi, R.A. (1856a) Plantarum novarum Chilensium, Centuria prima. Linnaea: Ein Journal für die Botanik in ihrem ganzen Umfange 28: 609-660.

Philippi, R.A. (1856b) Plantarum novarum Chilensium, Centuria secunda. Auctore eoden. Linnaea: Ein Journal für die Botanik in ihrem ganzen Umfange 28: 661-704.

Philippi, R.A. (1860) Florula Atacamensis seu Enumeratio Plantarum in itinere per desertum Atacamense observatarum. Eduard Anton, Halle, 62 pp.

Philippi, R.A. (1862a) Botánica. Sertum mendocinum. Catálogo de las plantas recojidas en Mendoza i en el camino entre ésta provincia i Chile por el portezuelo del Portillo, por don Wenceslao Diaz en los años 1860 i 1861. Anales de la Universidad de Chile 21: 389-407. http://dx.doi.org/10.5354/0365-7779.1862.2575

Philippi, R.A. (1862b) Botánica. Descripcion de unas plantas nuevas, recojidas en el verano pasado en la provincia del Maule i en Chillan por don Jerman Volckmann. Anales de la Universidad de Chile 21: 442-450. http://dx.doi.org/10.5354/0365-7779.1862.2576

Philippi, R.A. (1872) Botánica: descripcion de las plantas nuevas incorporadas últimamente en el herbario chileno. Anales de la Universidad de Chile 41: 663-746. http://dx.doi.org/10.5354/0365-7779.1872.23716

Philippi, R.A. (1891) Catalogus Praevius Plantarum in Itinere ad Tarapaca a Friderico Philippi Lectarum. Anales del Museo Nacional de Chile, Santiago de Chile 8: 1-94.

Philippi, R.A. (1892) Plantas nuevas chilenas de las familias Crucíferas, Bixáceas, Violáceas, Poligaléas (continuación). Anales de la Universidad de Chile 81: 329-347. http://dx.doi.org/10.5962/bhl.title.8592

Presl, K.B. (1844) Botanische Bemerkungen. G. Hasse, Prague, 154 pp. http://dx.doi.org/10.5962/bhl.title.280

Prina, A.O. (2001) Nuevas combinaciones en Menonvillea (Brassicaceae). Hickenia 3(26): 93-94.

Reiche, C. (1895) Estudios críticos sobre la flora de Chile (continuación): familia Crucíferas. Anales de la Universidad de Chile 90: 77-157.

Rollins, R.C. (1955) A revisionary study of the genus Menonvillea (Cruciferae). Contributions from the Gray Herbarium of Harvard University 177: 3-57.

Salariato, D.L. \& Al-Shehbaz I.A. (2012) Menonvillea. In: Anton A.M. \& Zuloaga F.O. (eds.) Flora Argentina: Brassicaceae, vol. 8. Estudio Sigma, Buenos Aires, pp. 101-105.

Salariato, D.L., Zuloaga, F.O. \& Al-Shehbaz, I.A. (2012) Morphometric studies and taxonomic delimitation in Menonvillea scapigera and related species (Cremolobeae: Brassicaceae). Plant Systematics and Evolution 298: 1961-1976. http://dx.doi.org/10.1007/s00606-012-0694-5

Salariato, D.L., Zuloaga, F.O. \& Al-Shehbaz, I.A. (2013) Molecular phylogeny of Menonvillea and recognition of the new genus Aimara (Brassicaceae: Cremolobeae). Taxon 62: 1220-1234. http://dx.doi.org/10.12705/626.6

Sandwith, N.Y. (1928) New Species from the Andes of Argentina: II. Bulletin of Miscellaneous Information Kew 1928: 107109. http://dx.doi.org/10.2307/4107700

Schulz, O.E. (1928) Die von O. Berninger, A. Hollermayer und besonders von E. Werdermann in Chile gesammelten Cruciferen. In: Werderman, E. (ed.) Beiträge zur Kenntnis der Flora von Chile. Notizblatt des Botanischen Gartens und Museums zu Berlin-Dahlem 10(95): 460-472. 
Schulz, O.E. (1936) Cruciferae. In: Engler, A. \& Harms, H. (eds) Die Natürlichen Pflanzenfamilien, vol 17B, 2nd ed. Verlag von Wilhelm Engelmann, Leipzig, pp 227-658.

Spegazzini C.L. (1902) Nova Addenda ad Floram Patagonicam. Anales del Museo Nacional de Buenos Aires 7(2): 135-308.

Steudel, E.G. (1841) Nomenclator botanicus, seu, Synonymia plantarum universalis: enumerans ordine alphabetico nomina atque synonyma, tum generica tum specifica, et a Linnaeo et a recentioribus de re botanica scriptoribus plantis phanerogamis imposita, Vol II. typis et sumptibus J.G. Cottae, Stuttgart \& Tübingen, 810 pp. http://dx.doi.org/10.5962/bhl.title.655

Steudel, E.G. (1856) Einige Beiträge zuder Chilesischen und Peruanischen Flora, hauptsächlich nach den Sammlnngen von Bertero und Lechler. Flora oder Botanische Zeitung: welche Recensionen, Abhandlungen, Aufsätze, Neuigkeiten und Nachrichten, die Botanik betreffend, enthält herausgegeben von der Königl. Botanischen Gesellschaft in Regensburg 39(26): 401-412.

Turczaninow, N.S. (1846) Generum Adhuc non Descriptorum, Adjectis Descriptionibus Nonnullarum Specierum Byttneriacearum. Bulletin de la Société impériale des naturalistes de Moscou 19(3): 497-510.

Turczaninow, N.S. (1854) Animadversiones ad Primam Partem Herbarii Turczaninowiani, Nunc Universitatis Caesareae Charkowiensis. Bulletin de la Société impériale des naturalistes de Moscou 27(4): 271-372.

Turczaninow, N.S. (1863) Animadversiones ad Catalogum Primum et Secundum Herbarii Universitatis Charkoviensis. Bulletin de la Société impériale des naturalistes de Moscou 36(2): 545-615.

Warwick, S.I., Mummenhoff, K., Sauder, C.A., Koch, M.A. \& Al-Shehbaz, I.A. (2010) Closing the gaps: Phylogenetic relationships in the Brassicaceae based on DNA sequence data of nuclear ribosomal ITS. Plant Systematics and Evolution 285: 209-232.

http://dx.doi.org/10.1007/s00606-010-0271-8

\section{Appendix I. Numerical List of Taxa and Specimens Examined}

\section{Numerical List of Taxa}

\section{Menonvillea Sect. Cuneata}

1. Menonvillea cicatricosa (Phil.) Rollins

2. Menonvillea comberi Sandwith

3. Menonvillea cuneata (Gillies \& Hook.) Rollins

4. Menonvillea frigida (Phil.) Rollins

5. Menonvillea macrocarpa (I.M. Johnst.) Rollins

6. Menonvillea nordenskjoeldii (Dusén) Rollins

7. Menonvillea patagonica Speg.

8. Menonvillea rigida Rollins

9. Menonvillea spathulata (Gillies \& Hook.) Rollins

10. Menonvillea virens (Phil.) Rollins

Menonvillea Sect. Menonvillea

11. Menonvillea chilensis (Turcz.) B.D. Jacks.

12. Menonvillea constitutionis (Phil.) Rollins

13. Menonvillea filifolia Fisch. \& C.A. Mey.

14. Menonvillea flexuosa Phil.

15. Menonvillea linearis DC.

16. Menonvillea litoralis (Barnéoud) Rollins

17. Menonvillea marticorenae (Al-Shehbaz) Salariato \& Al-Shehbaz

18. Menonvillea minima Rollins

19. Menonvillea orbiculata Phil.

20. Menonvillea pinnatifida Barnéoud

21. Menonvillea purpurea (Hastings) Rollins

\section{Menonvillea Sect. Scapigera}

22. Menonvillea famatinensis (Boelcke) Rollins

23.1. Menonvillea scapigera (Phil.) Rollins subsp. longipes (Rollins) Prina

23.2. Menonvillea scapigera (Phil.) Rollins subsp. scapigera

24. Menonvillea zuloagaensis Al-Shehbaz

Specimens Examined. Specimens are arranged by collector (with first initial, when known) in alphabetical order, followed by collector's number in increasing order (s.n. = without number), followed by species number in parentheses. 